Research Article

\title{
Expressway Surface Point Extraction from Mobile Laser Scanning Point Clouds
}

\author{
Rufei Liu*, Maoyi Tian, Bo Shi and Junyi Xu \\ Geomatics College, Shandong University of Science and Technology, Qingdao, China
}

Received 15 May 2013; Accepted 25 July 2013

\begin{abstract}
According to the problem between the cost of production, efficiency and data accuracy of expressway surface terrain data, this paper presents a fast ground point extraction method of expressway road surface from mobile laser scanning (MLS) point cloud data. Through the analysis of the spatial characteristics of MLS point cloud data in expressway, a triangle plane constraint (TPC) method is used to extract initial road surface points in the grid, and then multi-scale neighborhood iterative analysis (MNIA) method is proposed to further filter them, finally the high density road surface points are extracted from the original points based on neighborhood slope which is estimated by iterative least square within eight neighboring grid. Experiments are processed to verify the effectiveness of the algorithm by using two groups of actual point cloud data.
\end{abstract}

Keywords: Road surface extraction; MLS; Ground point filtering; MNIA

\section{Introduction}

As an advanced measurement method, MLS has the characteristics of fast, non-contact, real-time, dynamic, active, high density and high precision etc [1]. In the measurement of expressway, MLS can collect large area, high precision, high density surficial information of road surface, transport facilities, vegetation, road and affiliated objects. High-precision road terrain obtained by road point cloud data is basic data for further analysis of applications such as road surface settlement, pavement, slope collapse. And how to extract expressway terrain point cloud from the original MLS point cloud data quickly and accurately is the premise for these in-depth applications [2].

At present, many scholars have studied laser point cloud data. Axelsson[3] put forward an algorithm called adaptive TIN encryption to handle mechanical loading data in urban areas. Firstly, the algorithm created a sparse TIN based on a few seed points, and then TIN was constantly encrypted through iterative approach according to parameter thresholds. Vosselman[4] proposed the filter algorithm based on change of slope, the key of which was to select the appropriate slope threshold. He thought that slope thresholds should be chosen by prior knowledge of experimental area. The difficulty of the algorithm was increased because of the need for all the ground form samples. Different sizes of windows used to conduct a number of morphological filtering was advanced by Kilian[5]. The algorithm started from the minimum window size, and weight data of ground point was set according to the size of the window. With the gradual increase of window size and iterative processing, the bigger

\footnotetext{
*E-mail address: liurufei_2007@126.com

ISSN: 1791-2377 @ 2013 Kavala Institute of Technology. All rights reserved.
}

the window, the greater the weight is. Eventually, the terrain surface was calculated based on the weight of all the ground point. Zhang[6] described a progressive morphological filtering algorithm to filter feature points, which used mathematical morphology method to calculate the corresponding threshold based on terrain slope and window size. In the aspect of vehicle, Sherif[7] proposed a cluster analysis method based on point cloud spatial feature vector. It classified sidewalk, pavement and curbstone by calculating the normal direction and characteristic value. Anttoni[8] used intensity and elevation image to classify the road marking and curbstone automatically and built the road surface through delaunay TIN. Yang Bi-sheng[9] raised a method which extracted pavement and curbstone point from large-scale vehicle-borne laser point cloud by the scan-line one by one. A method of classifying vehicle-borne laser point data based on object features advanced by Li Ting[10] which realized terrain classification on the basis of object features summary. Yang Yang[11] put forward a vehicleborne Lidar point cloud filtering method base on scan lines. He divided scan lines into different segments according to slope difference.

The current MLS point cloud data processing researches mainly concentrate on the extraction of urban neighborhoods independent features, and in the aspect of road point cloud filtering, the pavement point was usually judgment only by height threshold, and did not consider the terrain features in different regions. Although more ground point filtering methods exist in the airborne ground point processing, the scanning distance, target, density and accuracy are different between the airborne point cloud processing and the MLS where the former is designed to extract a large area of continuous terrain, while the latter aims at the extraction of 
local high-precision, high-detail terrain, so the airborne approach is not applicable to the mobile point cloud data filtering. This paper tries to study the methods respectively suitable to the road surface through researches on the point cloud characteristics. In the aspect of road surface processing, such ideas as a three-dimensional virtual grid, MNIA and local slope filtering ideas are applied.

\section{Point cloud data preprocessing}

\subsection{Establishment of three-dimensional virtual grid}

To overcome the problems of low operation efficiency and information loss, this paper introduces a three-dimensional virtual grid to point cloud data processing [12]. When a virtual grid is established, as the data collected by expressway is in strip distribution, it will add redundant grids if the entire area is evenly split into grids. The MLS system records the vehicle track information, by which grids are established in strip distribution as shown in Figure 1(a), the red dotted lines indicates the lane tracks and the number of grids are significantly reduced. Grid size is determined by the scanning point density and terrain slope, for example, when the scanning point is dense and terrain slope is steep, a smaller grid step length should be set; while the scanning point is less dense and terrain slope is level, a relatively large grid step size should be set. Virtual grid is shown in Figure1(b) where the dot represents the point cloud and rectangular blocks represent the virtual grid.



(a)

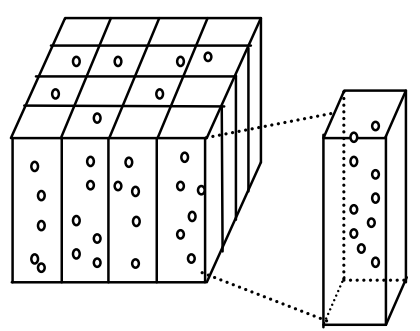

(b)

Fig.1. 3D virtual grid. (a) Plane distribution of 3D virtual grid. (b) 3D view of virtual grid

\subsection{Outliers removal}

In the data collection, due to the presence of random error, the original point cloud data unavoidably includes outliers which are needed to be removed before the filtering process $[13,14]$. As the MLS point cloud is the surface feature points in space, the point cloud is dense and the number of local spatial point cloud is more, while the outliers are generally mutational elevation points which are in discrete distribution with other points in the virtual grid as the red dot shown in Figure 2. So the statistical analysis can be conducted on the grid points within a number of different elevation interval layers. When the number is less than a certain threshold, the layer is an outlier elevation interval layer which should be removed.

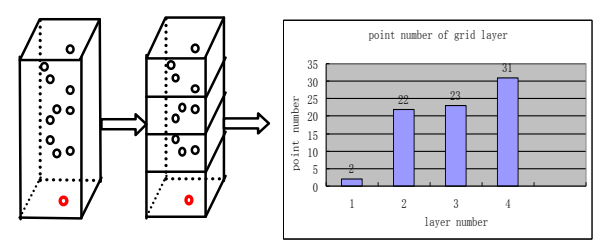

Fig.2. Principle diagram of grid statistical

\section{Expressway Surface Filtering Algorithm}

The ground point of MLS system is of high density where the average distance between points is less than $0.1 \mathrm{~m}$, average density of ground point is greater than 100 points/m2 and such point clouds can be used to generate highly accurate road surface topography and section of roads as well as can be used to check the subsidence and damage of the road surface. As the expressway surface is prone to appear small pothole damage which is around $0.15 \mathrm{~m}$, so instead of interpolated sampling, the surface filtering needs to extract all road surface laser point.

\subsection{Robust constraint of road surface initial point}

Expressway surface has smaller slope and point cloud is in regular distribution, Non-ground points mainly include transport facilities, vehicles and so on. In the threedimensional virtual grid of road surface, the lowest value of elevation of each grid point is usually considered as the initial ground grid point. To improve the robustness and probability of the initial ground point within the grid, this paper gets an initial ground point through TPC method. The principle is: Assuming that ground points exist within a single grid, as the local area of road surface(less than $1 \mathrm{~m} 2$ ) is close to horizontal with the same slope, then there should be a surface plane close to horizontal in this area, which need at least three non-collinear ground points that meet the following conditions: 1) the length of the triangle constituted by these three points should be greater than the distance threshold value ( $\mathrm{L}>1 / 2 * \mathrm{c}, \mathrm{c}$ is grid step length); 2 ) the height difference between any two points should be less than the threshold value ( $\mathrm{H}>\mathrm{HO}, \mathrm{HO}$ is height error of MLS); 3 ) the principle of optimal triangular shape should be followed and the triangle formed by three points should be close to an equilateral triangle. Virtual triangular plane constraint model grid is shown in Figure 3. All the points in the grid should be removed when there does not exist the three points that meet the above conditions.

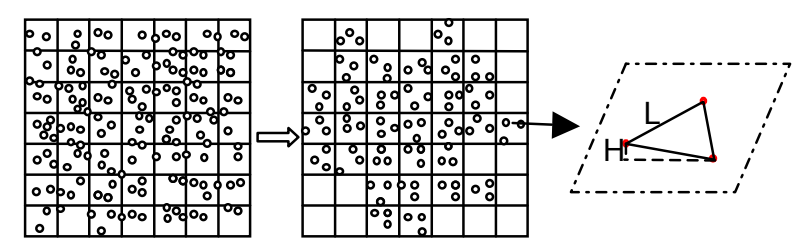

Fig.3. Ground point filtering by TPC

\subsection{Multi-scale neighborhood iterative analysis}

Although the probability of initial ground point is improved by triangle robust constraints, in data collection, due to the diversity of road surface features, when the flat terrain with larger area and almost horizontal to the surface is scanned, such surface features as low, neat surface vegetation, the grid low points will be incorrectly interpreted as ground 
point. To solve this problem, this paper designs MNIA method to do the multiple filtering for the target grid, further determining whether the low points within the grid are the road points. Algorithm process is as follows:

1) Estimating the neighborhood slope threshold. After the processing of triangle constraint, the lowest points of each grid are extracted from the target grid, and then the local area plane is fitted through least square, taking the plane slope as the local approximate gradient threshold [1516]. Because there may exist non-ground points among the initial lowest points, when using the least square method, the standard deviation of the distance(S) which is from each point to the fitting plane is calculated and the non-ground points are removed by comparison between $\mathrm{S}$ and standard deviation. The plane is fitted again to obtain more robust estimated value of the solid surface parameters (a, b, c). Specific procedures are as follows:

(a) The spatial plane equation of the local area is as follows, using the least square method to calculate the initial value $(a, b, c)$ of the plane;

$z=a x+b y+c$

(b) Based on the calculated value (a, b, c), using follow equation to calculate the distance(Si)from each point to the fitting plane;

$S_{i}=\frac{\left|a x_{i}+b y_{i}-z_{i}+c\right|}{\sqrt{a^{2}+b^{2}+1}}$

(c) Calculating the standard deviation $(\sigma)$ of the distance;

$$
\left\{\begin{array}{l}
\bar{S}=\frac{1}{n} \sum_{i=1}^{n} S_{i} \\
S=\left|\bar{S}-S_{i}\right| \\
\sigma=\sqrt{S^{T} P S /(n-1)}
\end{array}\right.
$$

Where $\mathrm{n}$ is the number of point, $\mathrm{P}$ is the unit matrix.

(d) When $\mathrm{Si}>2 \sigma$, this point is considered to be nonground point which should be removed. Conversely, should be retained;

(e) All the retained points are used to recalculate the parameters $(\mathrm{a}, \mathrm{b}, \mathrm{c})$ by applying the least square method again. Repeat the above steps, and the best plane parameters can be obtained if there are no points in need of removal.

2) Grid slope estimates and comparative analysis. As shown in Figure 4, firstly the unknown grid A are compared to the low points of 8 neighborhood grid. Striking triangular surface slope $[3,4,17]$, that is, the fanning triangle with $\mathrm{A}$ as its vertex composed by the lowest point of grid A and the 8 neighborhood grid, limiting the length of triangle side larger than half of grid step length and assuring it an acute triangle. The slope for each triangular face is calculated to the horizontal plane and comparing with the approximate slope threshold value. If each slope is smaller than the threshold, the lowest point is the ground point, otherwise, all the points within the grid are non-ground points that should be removed, and the grid is no longer involved in the next filtering step.

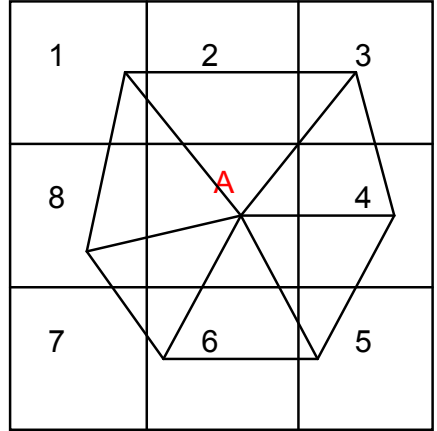

Fig.4. Principle diagram of slope estimation

3) Multi-scale iterative analysis. The premise of 8 neighborhood analysis is that at least one grid contains the ground point within the neighborhood. If the low points of grid $\mathrm{A}$ and its 8 neighboring grid are both non-ground points and have similar elevation, just as shown in Figure 5, the elevation of grid A and its around areas are all $2 \mathrm{~m}$ and the point in the grid are all non-ground. Under this condition, it is hard to remove the grid A using the 8 neighborhood methods. So this paper proposes Multi-scale iterative analysis method. Firstly, using the same neighborhood window to iterate the last results, and then expand the neighborhood analysis window, repeat iterative processing. The non-ground grid points of road surface and space isolated grid points are removed by multi-scale iteration until the difference between the numbers of reserved grid is zero.

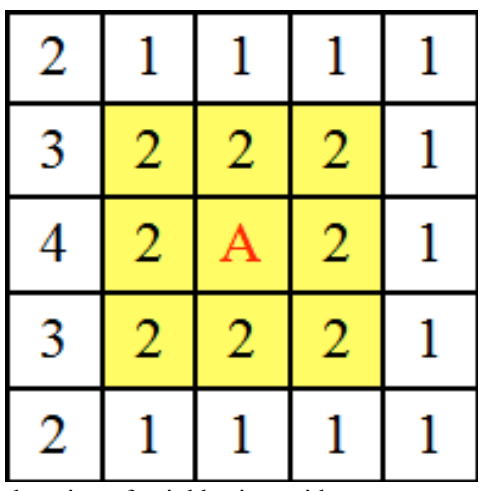

Fig.5. Similar elevation of neighboring grid

\subsection{Road surface point extraction within grid}

To improve the filtering accuracy of road point cloud, this paper limits the range of filter slope within grid 8 neighborhood, that is, the fanning triangle is constituted by the points to be determined within the grid and the 8 neighboring grid in accordance with the above grid slope estimation method. Calculating the slope of the triangle face and taking the maximum slope value to compare with the estimated threshold value of 8 neighborhoods, those within the threshold value range are the surface points.

\section{Experimental Studies}

In this paper, the above algorithm is realized by using c++, and two typical point cloud data which obtained by V-Surs I mobile 3D measurement system of Qingdao Xiu Shan Mobile Surveying corporation are selected to do the experimental analysis of noise removal and road surface filtering. The height error of MLS is about $2 \mathrm{~cm}$. Point cloud 
types in the area include roads, traffic ancillary facilities, vegetation and cars, where two sections of expressway are about $130 \mathrm{~m}$ length, the road surface slope is less than $4.5 \%$. The parameters of relevant data are shown in Table 1.

The statistical data of road extraction algorithm results are in Table 2. Grid horizontal step length and vertical direction are respectively $1 \mathrm{~m}^{*} 1 \mathrm{~m}^{*} 1 \mathrm{~m}$ when outliers is removed. According to the scanning point density and feature of space rod-shaped object, it is determined to be outlier grid when the point's number in grid is less than 10 points, and it is need to be removed. As a result, 2502 outlier points have been removed in straight road and 8685 outlier points have been removed in curve road. It can be known that the TPC and MNIA can effectively increase the probability of the initial low ground points and at the same time it can greatly reduce the grid number required for processing. 1962 non-ground grids of the straight road are removed by two step operation and 1365 non-ground grids of the curve road are removed.

\begin{tabular}{|c|c|c|c|c|c|c|}
\hline \multirow{2}{*}{$\begin{array}{c}\text { Experimental } \\
\text { road }\end{array}$} & \multirow{2}{*}{$\begin{array}{c}\text { Point } \\
\text { number }\end{array}$} & \multirow{2}{*}{$\begin{array}{c}\text { Length } \\
(\mathrm{m})\end{array}$} & \multicolumn{2}{|c|}{$\begin{array}{c}\text { Average point } \\
\text { distance }(\mathrm{m})\end{array}$} & $\begin{array}{c}\text { Road } \\
\text { surface } \\
\text { slope }(\%)\end{array}$ & $\begin{array}{l}\text { Biggest } \\
\text { elevation } \\
\text { difference } \\
(\mathrm{m})\end{array}$ \\
\cline { 5 - 7 } & & $\begin{array}{c}\text { Travel } \\
\text { direction }\end{array}$ & $\begin{array}{c}\text { Scanner } \\
\text { direction }\end{array}$ & & 8.06 \\
\hline Straight road & 837703 & 124 & $>0.11$ & $>0.027$ & $<4$ & 7.34 \\
\hline
\end{tabular}

\begin{tabular}{|c|c|c|c|c|c|c|c|c|}
\hline \multirow[t]{2}{*}{$\begin{array}{c}\text { Experimental } \\
\text { road }\end{array}$} & \multicolumn{2}{|c|}{$\begin{array}{l}\text { Outliers removal } \\
\qquad \text { ( point number) }\end{array}$} & \multicolumn{2}{|c|}{$\begin{array}{c}\text { TPC } \\
\text { (grid number) }\end{array}$} & \multicolumn{2}{|c|}{$\begin{array}{c}\text { MNIS } \\
\text { (grid number) }\end{array}$} & \multirow{2}{*}{$\begin{array}{c}\text { Road points } \\
\text { (point } \\
\text { number) }\end{array}$} & \multirow[t]{2}{*}{$\begin{array}{l}\text { Time } \\
\text { (s) }\end{array}$} \\
\hline & $\begin{array}{l}\text { Before } \\
\text { process }\end{array}$ & $\begin{array}{c}\text { After } \\
\text { process }\end{array}$ & $\begin{array}{l}\text { Before } \\
\text { process }\end{array}$ & $\begin{array}{c}\text { After } \\
\text { process }\end{array}$ & $\begin{array}{l}\text { Iterative } \\
\text { number }\end{array}$ & $\begin{array}{c}\text { After } \\
\text { process }\end{array}$ & & \\
\hline Straight road & 837703 & 835199 & 7246 & 5519 & 3 & 5284 & 609396 & 20 \\
\hline Curve road & 688924 & 680239 & 4952 & 2823 & 5 & 2587 & 458208 & 17 \\
\hline
\end{tabular}

The comparison before and after outliers removal of an experimentation area is in Figure 6 and the outlier points in red circle was successfully removed. The comparison before and after non-ground points removed by TPC and MNIA is shown in Figure7. We can get the massage that the nonground points exist on both road sides, and they are successfully removed by two step processing. Figure 8 and Figure 9 describe the result of road surface filtering of two road section, from which we can see that the point such as vegetation, traffic facilities and so on, are filtered exactly, and that the road shoulder part of the driveway can also be extracted. Road surface point cloud filtering proves that the algorithm achieves good filter results.

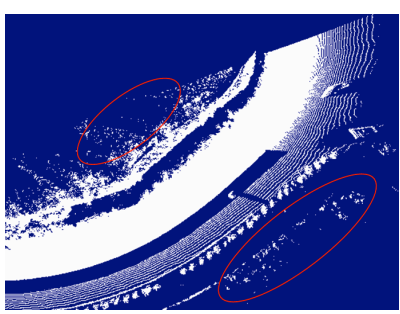

(a)

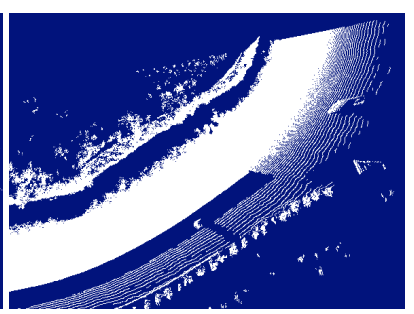

(b)
Fig.6. Comparison of before and after outliers removal. (a) Before outliers removal. (b) After outliers removal

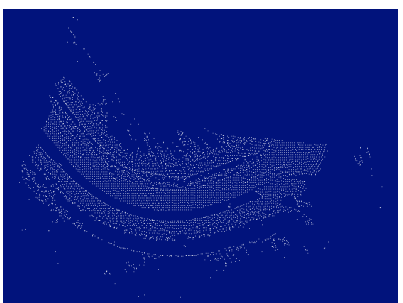

(a)

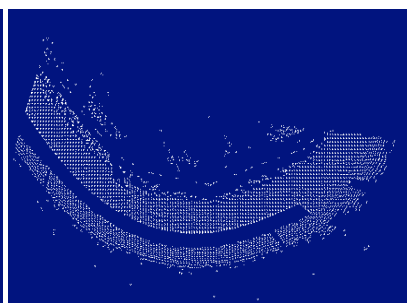

(b)

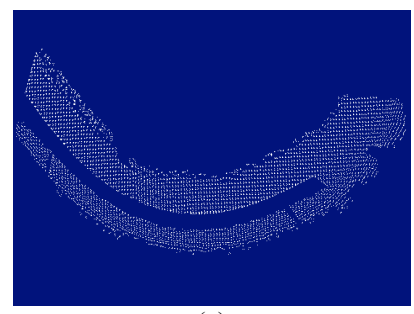

(c)

Fig.7. Comparison of before and after non-ground point filtering of road surface. (a) Original point. (b) After TPC filtering. (c) After MNIA filtering

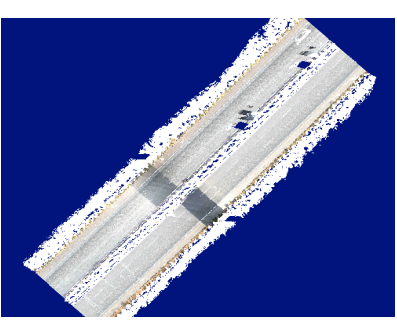

(a)

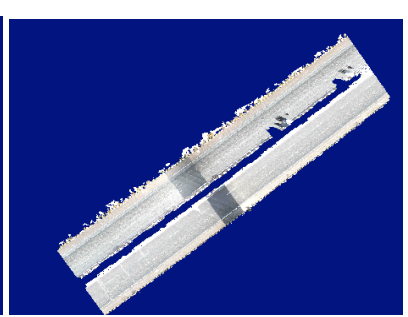

(b)
Fig.8. Filtering results of straight road. (a) Original point cloud data. (b) Straight road surface

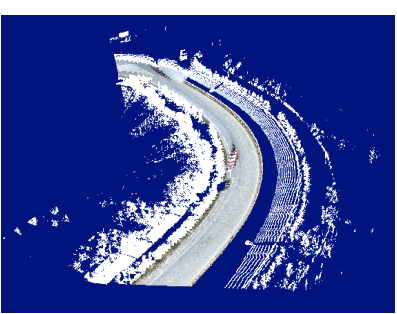

(a)

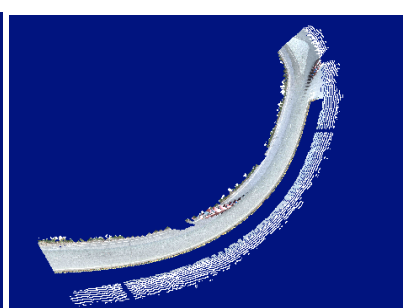

(b)
Fig.9. Filtering results of curve road. (a) Original point cloud data (driving on the left). (b) Curve road surface 


\section{Conclusions}

Point cloud data of expressway surface can be obtained by MLS measuring system, and in this paper, through the analysis of the spatial distribution characteristics of expressway point cloud data, we organize them by 3D virtual grid based on vehicle trajectory. The removal method of outliers is proposed. We focus on the study of the ground point filtering algorithm for the characteristics of expressway surface. Experimental results prove that the filtering method proposed in this paper has strong adaptability, high stability and can effectively extract the road high-density surface point clouds. In addition, the threshold parameter of algorithm requires some human experience and the adaptive threshold needs to be further researched.

\section{Acknowledgments}

This study is supported by the Funded by National Key Technology Research and Development Program of the Ministry of Science and Technology of China (2011BAK07B01), Graduate Innovation Fund Project of Shandong University of Science and Technology (YC130103), a Project of Shandong Province Higher Educational Science and Technology Program (J13LH04), Specialized Research Fund for the Doctoral Program of Higher Education(20113718120001) and Key Laboratory of Surveying and Mapping Technology on Island and Reef, NASG, China(2012A02). The authors thank anonymous reviewers for their constructive comments and suggestions.

Corresponding author: Maoyi Tian, Ph.D. and Associate Professor, E-mail: tianmaoyi_zhy@126.com

\section{References}

1. SHI,W.Z., LI,B.j.,LI,Q.Q.. A Method for Segmentation of Range Image Captured by Vehicle-borne Laserscanning Based on the Density of Projected Points. Acta Geodaetica et Cartographica Sinica, 2005,34(2),pp.95-100.

2. Li,Y.Q.; Sheng.Y.H.; Liu,H.Y.; Zhang,k.; Dai.H.Y. 3D road information extraction based on vehicle-borne laser scanning .Science of Surveying and Mapping,2008,33(4),pp.23-25.

3. Axelsson,P. DEM Generation from Laser Scanner Data Using Adaptive TIN Models.The International Archives of the Photogrammetry, Remote Sensing and Spatial Information Sciences. Annapolis, 2000, XXXIII(B3/1),pp.119-126.

4. Vosselman,G. Slope based filtering of laser altimetry data. The International Archives of the Photogrammetry, Remote Sensing and Spatial Information Sciences. Amsterdam, 1999, XXXIII (B3) ,pp.935-942.

5. Kilian ,J.; Haala ,N.; Englich ,M. Capture and Evaluation of Airborne Laser Scanner Data. IAPRS, 1996,31(B3) ,pp.383-388.

6. Zhang,K.Q.; et al. A progressive morphological filter for removing non-ground measurements from airborne LiDAR data.IEEE Transactions on Geoscience and Remote Sensing,2003,41( 4) ,pp. 872-882.

7. Sherif,E.H.*; Adel,M.; Derek,D. Lichti. Detection of road curb from mobile terrestrial laser scanner point cloud,2011 ISPRS Calgary 2011 Workshop, Vol.XXXVIII-5/W12, Calgary, Canada, 2011,pp.29-31

8. Anttoni Jaakkola; Juha H.; Hannu,H. Retrieval Algorithms for Road Surface Modelling Using Laser-Based Mobile Mapping. Sensors, 2008, 8,pp.5238-5249.
9. Bisheng,Y.; Lina F.; Jonathan L. Semi-automated extraction and delineation of 3D roads of street scene from mobile laser scanning point clouds.ISPRS Journal of Photogrammetry and Remote Sensing,2013, 79,pp.80-93.

10. Li,T.; Zhan,Q.M.; Yu,L. A Classification Method for Mobile Laser Scanning Data Based on Object Feature Extraction . Remote Sensing for Land \& Resources,2012,1,pp. 17-21.

11. Yang,Y.;Zhang,Y.S.; Ma,Y.W., et al. A Point Cloud Filtering Method of Vehicle-Borne LIDAR Based on Scanning Beam . Journal of Geomatics Science and Technology, 2010,27(3) ,pp.209212.

12. Zhang,H.; JIA,X.M.; Zhang,Y.S; et al. Filtering of Airborne LIDAR Data Based on Pseudo-Grid Concept and Modified Slope Filtering Algorithm. Journal of Geomatics Science and Technology,2009,26( 3),pp. 224-227.

13. Zuo,Z.Q.; Zhang ,Z.X.; Zhang,J.Q. Noise removal algorithm of LIDAR point clouds based on three-dimensional finite-element analysis . Journal of Remote Sensing, 2012, 16(2) ,pp.303-309.

14. Guan,Y.L.; Cheng,X.J.; Shi,G.G. A Robust Method for Fitting a Plane to Point Clouds . Journal of Tongji University(Natural Science),2008,36(7), pp.981-984.

15. Burkhard,S. A note on constrained total least squares estimation. Linear Algebra and its Application, 2006,(417),pp. 245-258.

16. Lu,T. D.; et al.Sphere target fixing of point cloud data based on TLS. Journal of Geodesy and Geodynamics,2009,29(4) ,pp. 102105.

17. Sithole,G. Filtering of laser altimetry data using a slope adaptive filter.International Archives of Photogrammetry and Remote Sensing,2001,34 ( 3 / W4 ),pp.203-210. 